\title{
Valorização de resíduos de ágata em argamassas e concretos: avaliação do ciclo de vida
}

\author{
Agate waste valorization in mortars and \\ concretes: life cycle assessment
}

\author{
Ana Carolina Passuello ${ }^{1}$, Simone Venquiaruto $^{2}$, Aguida Gomes Abreu ${ }^{1}$, \\ Camila Zanini ${ }^{1}$, Anilé Ossorio Dominguez ${ }^{1}$, Ana Paula Kirchheim ${ }^{1}$, \\ Denise Dal Molin ${ }^{1}$, Angela Masuero ${ }^{1}$
}

\footnotetext{
${ }^{1}$ Programa de Pós-Graduação em Eng. Civil: Construção e Infraestrutura - PPGCI/UFRGS, Porto Alegre, Rio Grande do Sul, Brasil.

${ }^{2}$ Universidade Federal do Pampa, Alegrete, Rio Grande do Sul, Brasil.

e-mail: ana.passuello@ufrgs.br, camila.zanini@ufrgs.br, aniossoriobrasil@gmail.com, aguidagabreu@gmail.com, anapaula.k@ufrgs.br,dmolin@ufrgs.br, angela.masuero@ufrgs.br, sivenquiaruto@gmail.com
}

\section{RESUMO}

O uso de resíduos oriundos do processo de beneficiamento de ágata (pó de ágata) em argamassas e concretos de cimento Portland é viável tecnicamente por suas propriedades mecânicas e de durabilidade. Porém, não existem pesquisas que avaliem o perfil ambiental dos materiais produzidos. O presente estudo visa avaliar os impactos ambientais potenciais associados ao ciclo de vida de argamassas e concretos fabricados com a utilização de pó de ágata como substitutivo do cimento Portland e de agregado miúdo, respectivamente. Para tanto, considerou-se proporcionamentos definidos em análises laboratoriais executadas previamente a este trabalho, em uma avaliação do berço ao portão (cradle to gate), onde foram avaliados os seguintes impactos, de acordo com a metodologia CML-IA: mudança climática, acidificação do solo e da água, eutrofização, depleção da camada de ozônio e depleção de recursos abióticos (fóssil e não fóssil). Resultados demonstram redução dos impactos potenciais de até $30 \%$, para o uso do resíduo em substituição ao cimento em argamassas. Reduções pouco expressivas são observadas para o uso do resíduo em substituição ao agregado miúdo, porém o ganho de resistência mecânica justifica a aplicação do resíduo em casos específicos. Uma vez que todos os impactos avaliados estão fortemente relacionados ao consumo de cimento, considera-se que o uso do resíduo em substituição ao cimento é a alternativa mais ambientalmente viável, para os casos avaliados. A participação dos processos relacionados ao transporte de mercadorias em todas as categorias de impacto valida a utilização de produtos produzidos/extraídos localmente. Finalmente, estudos futuros devem considerar o desempenho dos materiais produzidos, em comparação aos de referência.

Palavras-chave: Avaliação do Ciclo de Vida (ACV), resíduos, construção civil.

\section{ABSTRACT}

The application of residues from agate beneficiation process (agate powder) in mortars and concretes based on Portland cement is technically viable considering materials mechanical properties and durability. However, there is a lack of studies that assess the environmental profile of the produced materials. This paper aims to evaluate the potential environmental impacts associated to the life cycle of mortars and concretes produced with the application of agate powder in substitution of Portland cement and fine aggregate, respectively. To that, experimental designs defined in previous studies were considered, to perform a cradle to gate analysis, where the following impacts were evaluated, according to CML-IA method: climate change, soil and water acidification, eutrophication, ozone layer depletion, and resources depletion (fossil and non-fossil). Results show up to $30 \%$ reduction of potential impacts, for the application of the residue in substitution to Portland cement in mortars. Less expressive reductions are observed for the use of the residue in substitution of the fine aggregate; however, gains on mechanical strength justify the application of the residue in specific cases. Since all the assessed impacts are closely related to cement consumption, the application of the residue to replace cement is considered the most environmentally viable alternative, for the evaluated cases. The participation of the transport processes in all impact categories validates the application of materials locally produced/extracted. Finally, future studies shall consider the performance of the produced materials, in compari- 
son to the reference ones.

Keywords: Life Cycle Assessment (LCA), residues, civil construction.

\section{INTRODUÇÃO}

O aproveitamento de resíduos industriais como substituição do cimento ou dos agregados na produção de argamassas e concretos tem se mostrado, ao longo dos anos, muito benéfico. $\mathrm{O}$ uso desses resíduos pode melhorar as propriedades dos materiais, além de possibilitar um destino ambientalmente amigável a resíduos que, de outro modo, seriam dispostos em aterros industriais.

O Brasil é uma das nove regiões geográficas com excepcional volume de produção de gemas, destacando-se como o maior produtor de ágata do tipo Umbu [1]. O estado do Rio Grande do Sul (RS) é um dos mais importantes do País ao se tratar de pedras, gemas e joias [2], possuindo volume bastante expressivo de resíduo gerado pelo beneficiamento da ágata. Segundo Betat [3], o RS, no beneficiamento de pedras preciosas, como a ágata, gera resíduos que demandam um direcionamento para o passivo acumulado no processo, que hoje é depositado nos pátios das empresas. O pó de rejeito da indústria de beneficiamento de pedras preciosas, aqui denominado pó de rolagem, é um resíduo oriundo do processo do desgaste de fragmentos de rocha, a seco, em um gira moinho, com o propósito de obter pedras de forma arredonda, à semelhança de seixos, produzidas a partir de sobras de recortes de artefatos de rocha. A construção civil é uma alternativa para a utilização destes resíduos que, como consequência, traria uma diminuição do impacto ambiental e a contribuição para o desenvolvimento sustentável, o qual requer a redução do consumo de matérias primas naturais não renováveis.

Trabalhos anteriores [5-9] demonstram que o uso do pó de rolagem de ágata ou de seus fragmentos britados em argamassas e concretos de cimento Portland é viável tecnicamente do ponto de vista de seus aspectos no estado fresco (trabalhabilidade, consistência, entre outros), suas propriedades mecânicas e de durabilidade. Porém, não existem estudos que definam o perfil ambiental dos materiais produzidos.

Neste sentido, a Avaliação do Ciclo de Vida (ACV) realiza um balanço de todas as fases do ciclo de vida do produto em estudo - seja ele um insumo ou a edificação como um todo - suas interações, que podem ocorrer no tempo e no espaço, e seus impactos relacionados. A aplicação desta metodologia ao setor da construção civil é internacionalmente reconhecida, sendo previamente aplicada para o cálculo dos impactos do ciclo de vida de materiais de construção como o cimento [10,11], blocos cerâmicos [12], aberturas [13,14], pavimentos $[15,16,17,18]$, materiais isolantes [19] e estruturas em bambu [20]. Porém, os impactos relativos ao ciclo de vida de produtos e processos estão altamente relacionados a características locais do estudo. No Brasil, alguns estudos relacionados à aplicação do pensamento do ciclo de vida a materiais de construção já foram realizados. Saade et al. [21] e Stafford et al. [22] aplicaram a ACV para avaliação ambiental de cimentos produzidos no Brasil. Passuello el al. [23] compararam cimentos alternativos produzidos em laboratório de base Cálcio Sulfoaluminato (CSA) e geopolímeros [24] com Cimento Portland. Marques et al. [25] realizaram análise comparativa de tipos de fibras (polipropileno e de amianto) na produção de telhas. Ainda, Maia de Souza et al. [26] aplicaram a metodologia de ACV para a avaliação de sistemas de paredes. Porém, nenhum destes estudos avalia a produção de concretos e argamassas com incorporação de resíduos.

Neste contexto, insere-se o presente estudo, que visa avaliar os impactos ambientais potenciais associados ao ciclo de vida de argamassas e concretos fabricados com a utilização de resíduos oriundos do processo de beneficiamento de ágata (pó de rolagem) como substitutivo do cimento Portland e de agregado miúdo em concretos de cimento Portland.

\section{MATERIAIS E MÉTODOS}

A Avaliação do Ciclo de Vida (ACV) foi desenvolvida em quatro etapas: (1) definição de objetivo e escopo, (2) análise de inventário (ICV), (3) avaliação de impactos (AICV), e (4) interpretação.

\subsection{Definição do objetivo e escopo}

Esta ACV tem como objetivo definir o perfil ambiental de argamassas e concretos produzidos em laboratório a partir da substituição de cimento ou de agregado miúdo pelo resíduo de ágata (pó de rolagem). Para tanto, foram considerados os proporcionamentos dos materiais definidos experimentalmente [4, 8], e os materiais de construção disponíveis no local de estudo (Porto Alegre). Uma vez que os materiais possuem propriedades mecânicas distintas, não se pretende comparar os materiais produzidos, mas sim avaliar a possível melhoria em seu perfil ambiental decorrente da aplicação de diferentes teores de resíduo, em comparação às propriedades mecânicas observadas em estudos de laboratório. O sistema de produto em estudo consta na Fig.1. 


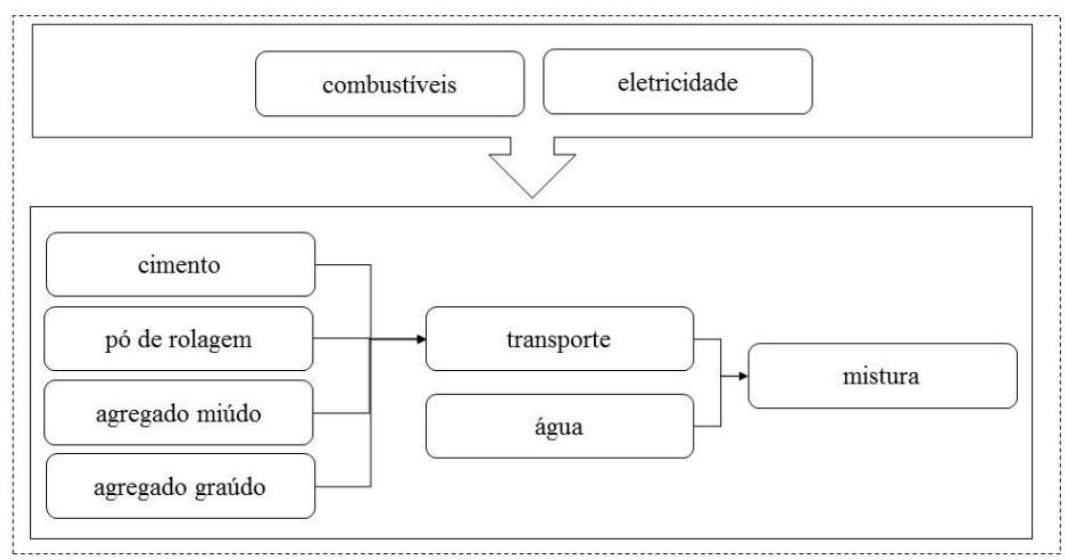

Figura 1: Fronteiras do sistema de produto em estudo, e a relação entre suas principais unidades de processo: produção do cimento, extração dos agregados, transporte de materiais ao canteiro de obra, produção de água tratada, mistura dos concretos e argamassas. Dados relativos à produção de combustíveis e eletricidade estão inseridos nas demais unidades de processo.

Estão considerados nas fronteiras do estudo a extração de matérias-primas (calcário para a produção do cimento, areia, brita basáltica), a produção do cimento, o transporte de materiais até o canteiro de obras, a energia da mistura. Não estão incluídos nas fronteiras do estudo a produção das fôrmas, o uso e disposição final dos materiais produzidos, uma vez que se considerou que estes seriam bastante similares dadas as características destes materiais. A unidade funcional definida é $1 \mathrm{~m}^{3}$ de material produzido (argamassa ou concreto), por se tratar da medida comercialmente utilizada.

Em relação ao transporte, considerou-se que a central de produção está localizada em Porto Alegre. Os materiais são transportados até a central de produção em caminhões com capacidade de 26 ton, com exceção do cimento que é primeiramente transportado da fábrica à central de distribuição por ferrovia, para a seguir ser transportado até a central de produção em caminhão. As distâncias entre os pontos de extração/produção de matérias-primas e a central de produção de argamassas e concretos, bem como os modais de transporte considerados, estão descritos na Tabela 1.

Tabela 1: Distâncias percorridas e modais utilizados.

\begin{tabular}{lcccl}
\hline Produto & Origem & Destino & Dist. $(\mathbf{k m})$ & Modal \\
\hline Cimento & Apiaí & Canoas & 900 & ferroviário \\
Cimento & Canoas & Porto Alegre & 15 & rodoviário \\
Ágata & Soledade & Porto Alegre & 225 & rodoviário \\
Areia & Porto Alegre & Porto Alegre & 10 & rodoviário \\
Brita & $\begin{array}{c}\text { Região metro- } \\
\text { politana }\end{array}$ & Porto Alegre & 100 & rodoviário \\
\hline
\end{tabular}

O resíduo de ágata utilizado (pó de rolagem) é proveniente da região de Soledade/RS e possui massa específica de $2,52 \mathrm{~g} / \mathrm{cm}^{3}$. A análise do pó de ágata por difração de raio X (DRX) evidenciou a predominância do mineral quartzo $(\mathrm{Q})$ na forma cristalina e, na análise por fluorescência de raio $\mathrm{X}$ (FRX), foi identificado sílica $\left(\mathrm{SiO}_{2}\right)$ em teores de $97,1 \%$, bem como a presença de $\mathrm{Al}_{2} \mathrm{O}_{3}(1,84 \%)$ e $\mathrm{Na}_{2} \mathrm{O}, \mathrm{K}_{2} \mathrm{O}$ e $\mathrm{CaO}$ em teores menores que $0,5 \%$. O resíduo, embora com valores muito próximos dos limites mínimos estabelecidos pela NBR 12653 [27] não foi classificado como material pozolânico. Foram realizados ensaios em argamassas com cal [28] e cimento Portland [29], no entanto, no ensaio com cal aos 7 dias, a resistência média dos corpos-de-prova foi de $5,1 \mathrm{MPa}$ (exige-se $\geq 6 \mathrm{MPa}$ ) e para as amostras com cimento a resistência média obtida foi $7 \%$ menor do que a referida norma estipula para o material ser considerado pozolânico. Assim, apesar do material não ser uma pozolana de acordo com a norma [27], ele apresenta uma reatividade não desprezível, o que levou à decisão de testar duas formas de utilização do resíduo: (a) como substituição, em massa, ao cimento, nos teores de $0,10,20$ e $30 \%$ de pó de rolagem passante na peneira de malha $0,15 \mathrm{~mm}$, produzindo-se argamassas e (b) pó de rolagem como substituição parcial, em volume (10 e 20\%), do agregado miúdo natural, produzindo-se concretos. 
Para a realização desta etapa da pesquisa, considerou-se o uso do cimento Portland de alta resistência inicial (CP-V ARI-RS). Como agregado, utilizou-se areia quartzosa proveniente do Rio Jacuí-RS e brita de origem basáltica oriunda de jazida da região. Conforme o fabricante, o cimento utilizado possui massa específica igual a $3,02 \mathrm{~g} / \mathrm{cm}^{3}$, superfície específica Blaine $4960 \mathrm{~cm}^{2} / \mathrm{g}$, finura na peneira de malha \# 200 igual a $0,1 \%$ e na peneira de malha \# 300 igual a $1,11 \%$.

Os agregados, areia de origem natural quartzosa e brita de origem basáltica, foram avaliados em relação à sua distribuição granulométrica, em acordo com NBR NM 248 [30] e NBR 7211 [31]. A distribuição granulométrica das areias sem e com a incorporação de pó de ágata, em teores de substituição de $10 \%$ e $20 \%$, em volume, resultou em composições de agregado miúdo com distribuição de tamanho de grãos dentro dos limites utilizáveis e módulo de finura igual a 2,21. A massa específica, determinada em acordo com a NBR

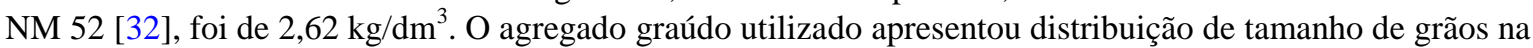
zona granulométrica d/D 9,5/25, dimensão máxima igual $25 \mathrm{~mm}$ e módulo de finura igual a 7,11. A massa específica, determinada em acordo com a norma NBR NM 52 [32], foi de $2,98 \mathrm{~g} / \mathrm{cm}^{3}$.

Selecionou-se, para o estudo em argamassas, quatro níveis de substituição de pó de rolagem e três traços unitários (1:M, aglomerante:areia), visando a obtenção de resultados na forma de diagrama de dosagem, para uma mesma consistência. Assim, mesmo em condições limitadas pelas relações água/aglomerante dos extremos, as curvas de Abrams possibilitam uma melhor visão conjunta do comportamento mecânico das misturas de argamassa, obtendo-se informações também relacionadas ao consumo de cimento e traços.

Nos concretos, a incorporação do pó de ágata deu-se em substituição à areia, em teores de 10 e $20 \%$, mantendo-se o abatimento. No estudo do concreto com pó de ágata, outro parâmetro estudado foi a relação água/cimento, definida a partir do concreto de referência, sem pó de rolagem, para três níveis de resistência à compressão.

\subsection{Inventário de Ciclo de Vida (ICV)}

Para a realização do ICV, dados primários foram obtidos em laboratório, através do proporcionamento dos materiais (Tabelas 2 e 3). Cabe salientar que, no caso das argamassas, as substituições foram realizadas em massa, enquanto que para os concretos as substituições da areia por pó de rolagem foram realizadas em volume. Foram dosados traços para atingir três níveis de resistência (ou relação a/c), tanto para os concretos como para as argamassas, ou seja, traços ricos $(\mathrm{R})$, médios $(\mathrm{M})$ e pobres $(\mathrm{P})$ frente à quantidade de cimento, sendo os traços R com maior consumo de cimento, e, consequentemente, menor relação a/c e maior resistência. Salienta-se que, na elaboração dos traços pobres das argamassas com substituição do cimento por pó de rolagem, foram necessários ajustes na proporção de agregados e água utilizados, resultando em traços mais pobres que os inicialmente previstos. Os traços finais obtidos e as quantidades de material cimentante utilizadas são apresentados na tabela 2 .

Tabela 2: Proporcionamento das argamassas

\begin{tabular}{|c|c|c|c|c|c|c|c|}
\hline $\begin{array}{l}\text { Teor de } \\
\text { pó } \\
(\%)\end{array}$ & Traço & $\begin{array}{r}\text { Traço unitário } \\
\text { (cim:areia:a/agl) }\end{array}$ & $\mathbf{a} / \mathbf{c}$ & $\begin{array}{r}\text { Cimento } \\
\left(\mathrm{kg} \mathrm{m}^{-3}\right)\end{array}$ & $\begin{array}{c}\text { Consumo } \\
\text { pó de } \\
\text { rolagem } \\
\left(\mathrm{kg} \mathrm{m}^{-3}\right)\end{array}$ & $\begin{array}{c}\text { Consumo } \\
\text { areia } \\
\left(\mathrm{kg} \mathrm{m}^{-3}\right)\end{array}$ & $\begin{array}{c}\text { Consumo } \\
\text { água } \\
\left(\mathrm{kg} \mathrm{m}^{-3}\right)\end{array}$ \\
\hline \multirow[t]{3}{*}{0} & OR & $1,00: 2,65$ & 0,53 & 517 & 0 & 1370 & 272 \\
\hline & $0 \mathrm{M}$ & $1,00: 3,65$ & 0,63 & 410 & 0 & 1497 & 258 \\
\hline & $\mathrm{OP}$ & $1,00: 4,65$ & 0,77 & 336 & 0 & 1562 & 257 \\
\hline \multirow[t]{3}{*}{10} & $10 \mathrm{R}$ & $0,90: 2,65$ & 0,53 & 464 & 52 & 1366 & 271 \\
\hline & $10 \mathrm{M}$ & $0,90: 3,65$ & 0,63 & 368 & 41 & 1493 & 258 \\
\hline & $10 \mathrm{P}$ & $0,90: 5,48$ & 0,77 & 265 & 29 & 1614 & 250 \\
\hline \multirow[t]{3}{*}{20} & $20 \mathrm{R}$ & $0,80: 2,65$ & 0,53 & 411 & 103 & 1361 & 271 \\
\hline & $20 \mathrm{M}$ & $0,80: 3,65$ & 0,63 & 327 & 82 & 1492 & 257 \\
\hline & $20 \mathrm{P}$ & $0,80: 5,48$ & 0,77 & 235 & 59 & 1610 & 250 \\
\hline \multirow[t]{3}{*}{30} & $30 \mathrm{R}$ & $0,70: 2,65$ & 0,53 & 359 & 154 & 1359 & 270 \\
\hline & $30 \mathrm{M}$ & $0,70: 3,65$ & 0,63 & 285 & 122 & 1486 & 256 \\
\hline & $30 \mathrm{P}$ & $0,70: 5,50$ & 0,77 & 204 & 88 & 1603 & 251 \\
\hline
\end{tabular}


Dados secundários foram retirados de bases de dados internacionais [33] (PE International, 2014), através da seleção de dados adaptados à realidade brasileira, disponíveis nesta base de dados. Para a construção do ICV e análise dos impactos ambientais, foi utilizado o software GaBi Professional v. 6.

Tabela 3: Proporcionamento dos concretos

\begin{tabular}{|c|c|c|c|c|c|c|c|c|}
\hline $\begin{array}{c}\text { Teor de pó } \\
(\%)\end{array}$ & Traço & $\begin{array}{l}\text { Traço unitário } \\
\text { (cim:areia:brita) }\end{array}$ & $\begin{array}{l}\mathbf{a} / \mathbf{c} \\
-\end{array}$ & $\begin{array}{c}\text { Consumo } \\
\text { cimento } \\
\left(\mathrm{kg} \mathrm{m}^{-3}\right)\end{array}$ & $\begin{array}{c}\text { Consumo } \\
\text { areia } \\
\left(\mathrm{kg} \mathrm{m}^{-3}\right)\end{array}$ & $\begin{array}{l}\text { Consumo } \\
\text { pó de } \\
\text { rolagem } \\
\left(\mathbf{k g ~ m}^{-3}\right)\end{array}$ & $\begin{array}{c}\text { Consumo } \\
\text { brita } \\
\left(\mathrm{kg} \mathrm{m}^{-3}\right)\end{array}$ & $\begin{array}{c}\text { Consumo } \\
\text { água } \\
\left(\mathrm{kg} \mathrm{m}^{-3}\right)\end{array}$ \\
\hline \multirow[t]{3}{*}{0} & OR & $1,00: 1,60: 2,40$ & 0,42 & 452 & 724 & 0 & 1085 & 190 \\
\hline & $0 \mathrm{M}$ & $1,00: 2,54: 3,26$ & 0,57 & 331 & 840 & 0 & 1078 & 188 \\
\hline & OP & $1,00: 3,78: 4,42$ & 0,77 & 243 & 920 & 0 & 1076 & 187 \\
\hline \multirow[t]{3}{*}{10} & $10 \mathrm{R}$ & $1,00: 1,60: 2,40$ & 0,42 & 452 & 651 & 70 & 1085 & 190 \\
\hline & $10 \mathrm{M}$ & $1,00: 2,54: 3,26$ & 0,57 & 331 & 756 & 81 & 1078 & 188 \\
\hline & $10 \mathrm{P}$ & $1,00: 3,78: 4,42$ & 0,77 & 243 & 828 & 88 & 1076 & 187 \\
\hline \multirow[t]{3}{*}{20} & $20 \mathrm{R}$ & $1,00: 1,60: 2,40$ & 0,42 & 452 & 579 & 139 & 1085 & 190 \\
\hline & $20 \mathrm{M}$ & $1,00: 2,54: 3,26$ & 0,57 & 331 & 672 & 162 & 1078 & 188 \\
\hline & $20 \mathrm{P}$ & $1,00: 3,78: 4,42$ & 0,77 & 243 & 736 & 177 & 1076 & 187 \\
\hline
\end{tabular}

\subsection{Avaliação de Impactos do Ciclo de Vida (AICV)}

Para o presente estudo, foram selecionadas as categorias indicadas pela norma inglesa EN 15804 [34], que aconselha o uso dos fatores de classificação do CML versão IA: mudança climática, acidificação do solo e da água, eutrofização, depleção do ozônio estratosférico e depleção de recursos abióticos (fóssil e não fóssil). Apesar da categoria ozônio fotoquímico ser também indicada por dita norma, esta não foi considerada devido à inexistência de uma ferramenta representativa da realidade local, uma vez que seus fatores de caracterização foram desenvolvidos com base nas emissões de NOx na Europa [35].

\subsection{Interpretação dos resultados}

Para a interpretação dos resultados, além dos valores de impactos calculados, considerou-se também a resistência mecânica dos materiais estudados. Uma vez que os materiais avaliados possuem propriedades diversas, não é possível a comparação direta dos mesmos. Porém, é importante que se avalie a variação na resistência mecânica para compreender como as propriedades mecânicas dos materiais podem ser afetadas com a aplicação do resíduo em diferentes teores. Os ensaios mecânicos foram realizados após o encerramento do período de cura dos concretos e argamassas, aos 28 dias. Neste caso, aplicou-se o ensaio de resistência à compressão axial, conforme as prescrições das normas NBR 5739 [36] e NBR 7215 [37], para concretos e argamassas respectivamente.

\section{RESULTADOS}

A continuação, são apresentados e discutidos os resultados dos impactos ambientais potenciais, para as argamassas e concretos avaliados, considerando as contribuições de cada uma das etapas do seu ciclo de vida.

\subsection{Impactos do ciclo de vida da produção de argamassas}

Os impactos ambientais potenciais das argamassas avaliadas estão apresentados na Tabela 4. Já a participação de cada unidade de processo nos resultados para o traço OR é apresentada na Figura 2. 
Tabela 4: Impactos ambientais potenciais por $\mathrm{m}^{3}$ de argamassa. Impactos avaliados: mudança climática (MC), acidificação do solo e da água (PA), eutrofização (PE), depleção da camada de ozônio (DCO) e depleção de recursos abióticos fósseis (DRA-F) não fósseis (DRA-E).

\begin{tabular}{lrrrrrr} 
& $\begin{array}{r}\text { MC } \\
\mathrm{kg} \mathrm{CO}_{2} \text {-eq }\end{array}$ & $\begin{array}{r}\text { PA } \\
\mathrm{kg} \mathrm{SO}_{2} \text {-eq }\end{array}$ & $\begin{array}{r}\text { PE } \\
\mathrm{kg} \mathrm{PO}_{3}^{-4} \text {-eq. }\end{array}$ & $\begin{array}{r}\text { DRA-F } \\
\text { MJ }\end{array}$ & $\begin{array}{r}\text { DCO } \\
\text { kg R11-eq. }\end{array}$ & $\begin{array}{r}\text { DRA-E } \\
\text { kg Sb-eq. }\end{array}$ \\
\hline $0 \mathrm{R}$ & 594 & 1,39 & 0,17 & 3532 & $6,73 \mathrm{E}-10$ & $7,11 \mathrm{E}-04$ \\
$10 \mathrm{R}$ & 535 & 1,26 & 0,16 & 3191 & $6,15 \mathrm{E}-10$ & $6,38 \mathrm{E}-04$ \\
$20 \mathrm{R}$ & 475 & 1,13 & 0,14 & 2849 & $5,58 \mathrm{E}-10$ & $5,65 \mathrm{E}-04$ \\
$30 \mathrm{R}$ & 417 & 1,00 & 0,13 & 2515 & $5,01 \mathrm{E}-10$ & $4,94 \mathrm{E}-04$ \\
$0 \mathrm{M}$ & 474 & 1,12 & 0,14 & 2839 & $5,69 \mathrm{E}-10$ & $5,64 \mathrm{E}-04$ \\
$10 \mathrm{M}$ & 427 & 1,01 & 0,13 & 2568 & $5,22 \mathrm{E}-10$ & $5,07 \mathrm{E}-04$ \\
$20 \mathrm{M}$ & 381 & 0,91 & 0,12 & 2304 & $4,77 \mathrm{E}-10$ & $4,50 \mathrm{E}-04$ \\
$30 \mathrm{M}$ & 334 & 0,80 & 0,10 & 2033 & $4,30 \mathrm{E}-10$ & $3,92 \mathrm{E}-04$ \\
$0 \mathrm{P}$ & 391 & 0,93 & 0,12 & 2357 & $4,95 \mathrm{E}-10$ & $4,63 \mathrm{E}-04$ \\
$10 \mathrm{P}$ & 311 & 0,75 & 0,10 & 1899 & $4,21 \mathrm{E}-10$ & $3,65 \mathrm{E}-04$ \\
$20 \mathrm{P}$ & 277 & 0,67 & 0,09 & 1694 & $3,79 \mathrm{E}-10$ & $3,24 \mathrm{E}-04$ \\
$30 \mathrm{P}$ & 243 & 0,59 & 0,08 & 1504 & $3,52 \mathrm{E}-10$ & $2,81 \mathrm{E}-04$ \\
\hline
\end{tabular}

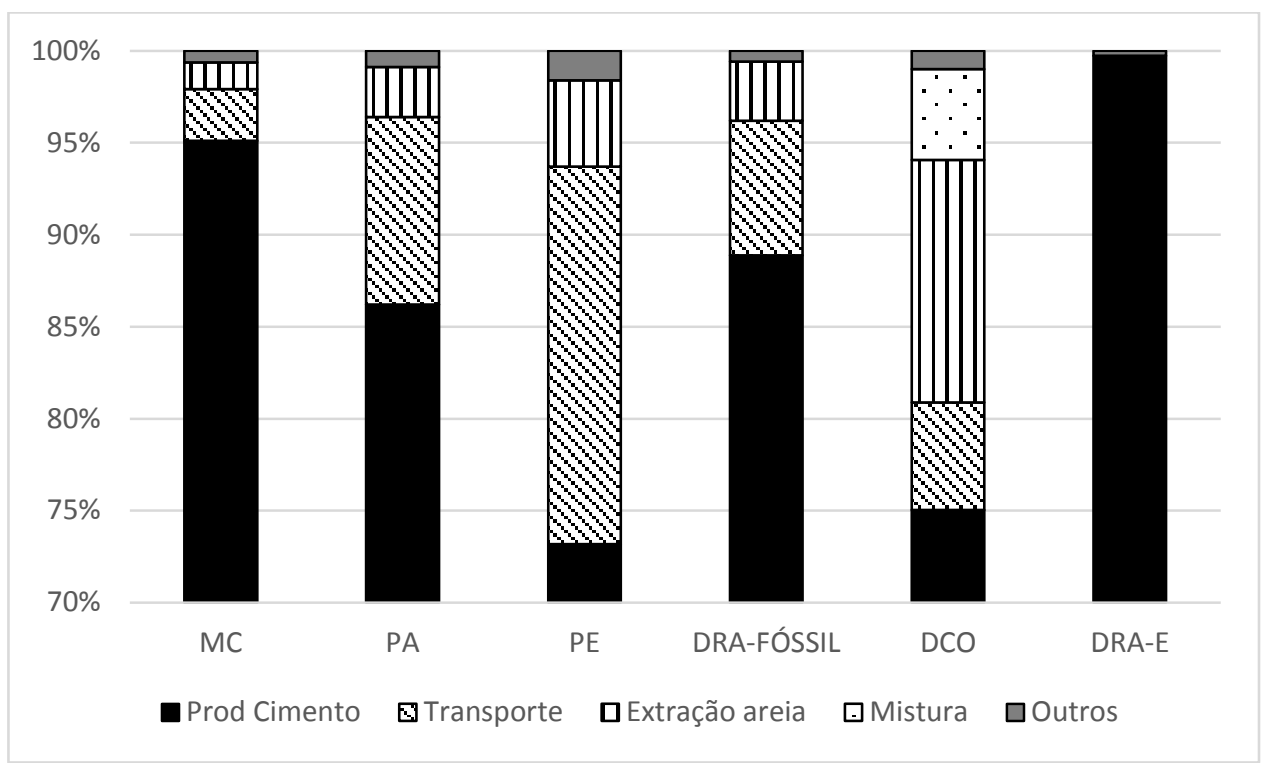

Figura 2: Participação de cada unidade de processos avaliada nos impactos do ciclo de vida da argamassa 0R. Impactos avaliados: mudança climática (MC), acidificação do solo e da água (PA), eutrofização (PE), depleção da camada de ozônio (DCO) e depleção de recursos abióticos fósseis (DRA-F) não fósseis (DRA-E).

Observa-se que os impactos ambientais potenciais estão fortemente relacionados ao consumo de cimento destes materiais (Figura 2), sendo a contribuição do processo de produção do cimento superior a $70 \%$ para todas as categorias avaliadas. Por isso, para cada um dos teores de substituição, as argamassas ricas apresentam um maior impacto em todas as categorias quando comparadas às argamassas médias e pobres.

Os impactos de mudança climática variam entre 243 e $594 \mathrm{~kg} \mathrm{CO}_{2}$-eq m${ }^{-3}$ argamassa. A produção de cimento é a etapa do ciclo de vida mais relevante no caso de mudanças climáticas, representando $95 \%$ do impacto total desta categoria para a argamassa 0R. O fator mais importante na produção de cimentos é a elevada emissão de dióxido de carbono característica de seu processo produtivo. No caso das argamassas ricas sem substituição, por exemplo, dos $594 \mathrm{~kg} \mathrm{CO}_{2}$-eq m m $^{-3}$ argamassa, 548 são relacionados às emissões de $\mathrm{CO}_{2}$ na produção de cimento. Deste modo, argamassas com maior teor de substituição apresentam maior redução dos valores desta categoria de impacto (até 30\% para argamassas ricas e médias e de 39\% para argamassas 
pobres, quando comparadas à referência). A redução de impacto entre o traço rico sem substituição (0R) e o traço pobre de maior substituição (30P) é de 60\%. Cabe destacar, porém, que as argamassas ricas, médias e pobres não cumprem a mesma função, uma vez que é esperado que o desempenho mecânico e durabilidade destes materiais apresente variações nos diferentes traços avaliados [9].

Os impactos potenciais de acidificação e eutrofização apresentam tendência similar, porém com menor participação do processo produtivo do cimento ( $86 \%$ para acidificação e $73 \%$ para eutrofização, Figura 2). A pronunciada participação do setor cimenteiro nesses casos se deve às emissões atmosféricas de NOx (ambos casos) e $\mathrm{SO}_{2}$ (acidificação). Destaca-se, no caso da eutrofização, a participação do transporte, representando $21 \%$ do PE para a argamassa 0R, graças à emissão de óxidos de nitrogênio (NOx) durante o transporte de mercadorias por trem, além das emissões de nitratos aos corpos d'água na produção de diesel.

$\mathrm{O}$ transporte de materiais também apresenta participação significativa nos impactos de mudança climática $\left(3 \%\right.$, associada à emissão de $\left.\mathrm{CO}_{2}\right)$ e acidificação $(10 \%$, emissão de NOx). Assim, a redução da distância entre os fornecedores e a central de produção (especialmente o de cimento, que se encontra a aproximadamente $900 \mathrm{~km}$ ) acarretaria em diminuição pronunciada destes impactos. Também, o uso de veículos de baixa emissão e modais mais eficientes poderia reduzir estes impactos.

No caso da depleção de recursos abióticos fósseis, mais uma vez a maior participação é a do processo produtivo do cimento (89\%), uma vez que este demanda importantes quantidades de combustíveis fósseis para operação dos fornos. É necessário um consumo de combustíveis fósseis entre 1464 e 3532 MJ para a produção de $1 \mathrm{~m}^{3}$ das argamassas avaliadas. Neste caso, também é bastante pronunciada a participação do transporte das matérias-primas $(7 \%)$ e a extração de areia (entre 3\%). Como se observa na Figura 3, as demandas de combustíveis para o transporte das matérias-primas variam de acordo com os teores de substituição. À medida que os teores de substituição aumentam, é necessária uma menor quantidade de cimento, diminuindo a necessidade de transporte ferroviário. Ao mesmo tempo, é incrementada a participação do transporte rodoviário. Apesar do transporte rodoviário consumir mais combustíveis por $\mathrm{kg} . \mathrm{km}$ de mercadoria transportada, a distância dos produtores de ágata é bastante inferior ao de cimento, no estudo de caso avaliado, fazendo com que a substituição seja positiva nesses casos.

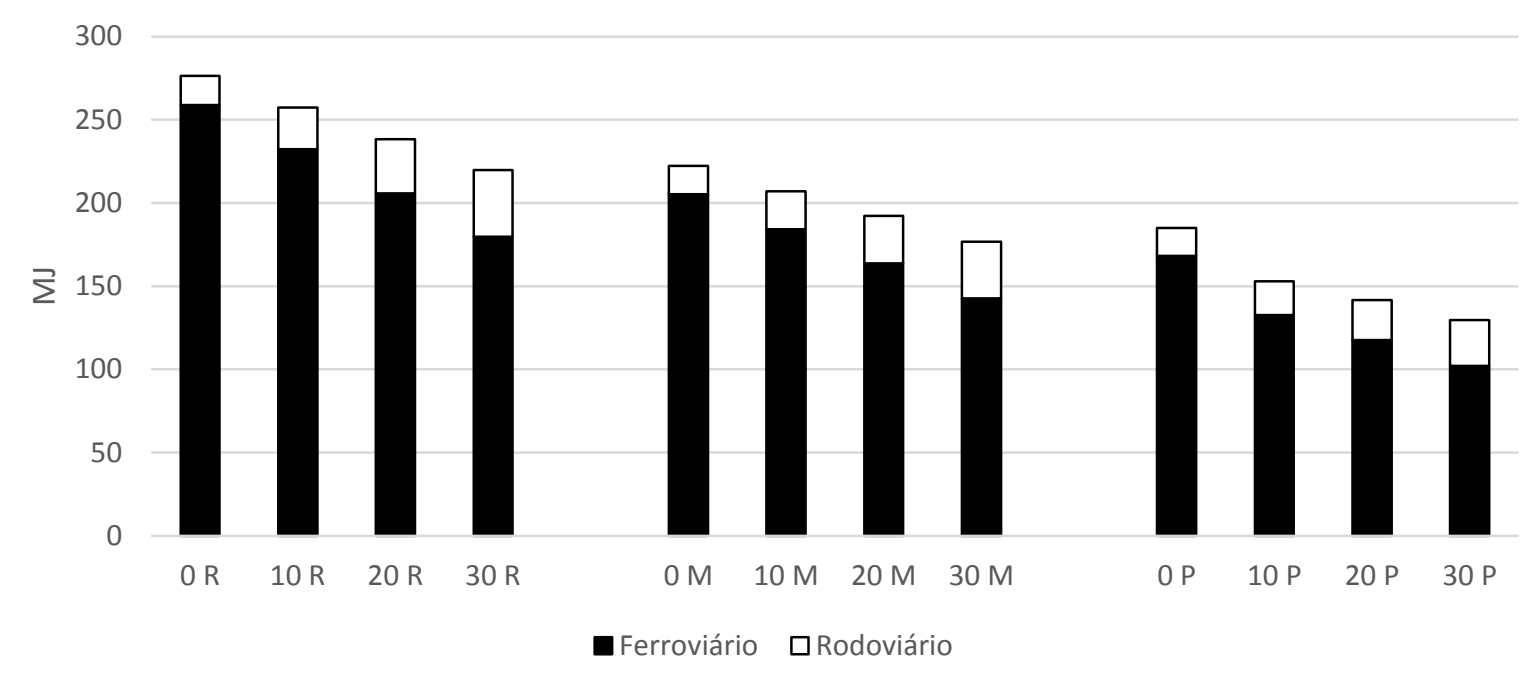

Figura 3: Demanda de combustíveis fósseis para o transporte de materiais necessários para a produção de $1 \mathrm{~m}^{3}$ de argamassa.

Para o caso da depleção da camada de ozônio, a participação da produção do cimento é menos pronunciada (75\%). Nota-se uma maior participação da extração de areia (13\%), do processo de mistura da argamassa (5\%), e do transporte (6\%). O impacto de depleção da camada de ozônio para os processos citados está relacionado à emissão de contaminantes orgânicos voláteis não-metânicos (NMVOC) ao ar.

Finalmente, para o caso da depleção dos recursos abióticos - não fósseis, apenas a produção do cimento aparece como consumidor relevante de recursos naturais, pelo uso da gipsita (Figura 2). Cabe destacar que a extração do calcário e da areia não estão contempladas na ferramenta de impacto selecionada [38].

\subsection{Impactos do ciclo de vida da produção de concretos}

Enquanto os impactos do ciclo de vida para a produção de $1 \mathrm{~m}^{3}$ de concreto nos diferentes proporcionamentos 
estão apresentados na Tabela 5, as participações de cada unidade de processo nos impactos do traço referência (0R) estão apresentados na Figura 4. De forma similar ao que se observa nas argamassas, os impactos ambientais da produção de concretos estão relacionados à produção do cimento (Figura 4), com participação entre 38 e $99 \%$ para as categorias de impactos avaliadas. Entretanto, no caso das argamassas, o resíduo de ágata foi utilizado em substituição ao cimento, enquanto que nos concretos este foi utilizado em substituição ao agregado miúdo. Por este motivo, o uso de diferentes teores de substituição não está relacionado à redução dos impactos (Tabela 5).

Os impactos de mudança climática variam entre 298 e $531 \mathrm{~kg} \mathrm{CO}_{2}$-eq m${ }^{-3}$ concreto. Este resultado comprova a correlação deste impacto com o consumo de cimento, tal como descrito na literatura. Knoeri et al. [39] realizaram um estudo em concretos estruturais produzidos com agregados reciclados e encontraram valores entre 240 e $250 \mathrm{~kg} \mathrm{CO}_{2}$-eq m${ }^{-3}$ concreto para consumos de cimento de $300 \mathrm{~kg} \mathrm{~m}^{-3}$; e $300 \mathrm{~kg} \mathrm{CO}_{2}$-eq m para consumos de cimento de $360 \mathrm{~kg} \mathrm{~m}^{-3}$. Neste estudo, para um consumo de cimento de $331 \mathrm{~kg} \mathrm{~m}^{-3}$ (traços $\mathrm{M}$ ), foram encontrados valores entre 396 e $397 \mathrm{~kg} \mathrm{CO}_{2}$-eq m${ }^{-3}$ concreto, um pouco superiores aos reportados pelos autores. Cabe destacar, entretanto, que os limites dos sistemas de estudo são diferentes, uma vez que Knoeri et al. [39] consideram os impactos evitados na reutilização de resíduos (evitando sua disposição em aterros e a extração de recursos naturais), o que é a provável justificativa da redução deste impacto. O processo mais relevante é, mais uma vez, a produção do cimento (93\%). Em comparação ao traço rico, os traços médio e pobre apresentam uma redução de emissões de gases do efeito estufa de 25 e $44 \%$, respectivamente (Tabela 5). Também deve ser mencionada a participação dos processos extração da brita (3\%), e transporte $(3 \%)$.

Tabela 5: Comparação dos resultados de AICV para os concretos avaliados. Impactos ambientais potenciais por $\mathrm{m}^{3}$ de concreto. Impactos avaliados: mudança climática (MC), acidificação do solo e da água (PA), eutrofização (PE), ozônio fotoquímico (DCO) e depleção de recursos abióticos fósseis (DRA-F) não fósseis (DRA-E).

\begin{tabular}{|c|c|c|c|c|c|c|}
\hline & $\begin{array}{r}\text { MC } \\
\mathrm{kg} \mathrm{CO}_{2} \text {-eq } \\
\end{array}$ & $\begin{array}{r}\text { PA } \\
\mathrm{kg} \mathrm{SO}_{2} \text {-eq } \\
\end{array}$ & $\begin{array}{r}\mathbf{P E} \\
\mathrm{kg} \mathrm{PO}_{3}{ }^{-4}- \\
\text { eq. }\end{array}$ & $\begin{array}{r}\text { DRA- } \\
\text { FÓSSIL } \\
\mathrm{MJ}\end{array}$ & $\begin{array}{r}\text { DCO } \\
\text { kg R11-eq. }\end{array}$ & $\begin{array}{r}\text { DRA-E } \\
\text { kg Sb-eq. }\end{array}$ \\
\hline OR & 531 & 1,27 & 0,16 & 3196 & $1,16 \mathrm{E}-09$ & $6,24 \mathrm{E}-04$ \\
\hline $10 \mathrm{R}$ & 531 & 1,28 & 0,16 & 3202 & $1,16 \mathrm{E}-09$ & $6,24 \mathrm{E}-04$ \\
\hline $20 \mathrm{R}$ & 531 & 1,28 & 0,16 & 3208 & $1,16 \mathrm{E}-09$ & $6,24 \mathrm{E}-04$ \\
\hline $0 \mathrm{M}$ & 396 & 0,97 & 0,13 & 2429 & 1,04E-09 & $4,58 \mathrm{E}-04$ \\
\hline $10 \mathrm{M}$ & 397 & 0,98 & 0,13 & 2436 & $1,04 \mathrm{E}-09$ & $4,58 \mathrm{E}-04$ \\
\hline $20 \mathrm{M}$ & 397 & 0,99 & 0,13 & 2443 & $1,03 \mathrm{E}-09$ & $4,58 \mathrm{E}-04$ \\
\hline $\mathrm{OP}$ & 298 & 0,75 & 0,10 & 1872 & $9,57 \mathrm{E}-10$ & $3,37 \mathrm{E}-04$ \\
\hline $10 \mathrm{P}$ & 299 & 0,76 & 0,10 & 1880 & $9,51 \mathrm{E}-10$ & $3,37 \mathrm{E}-04$ \\
\hline $20 \mathrm{P}$ & 299 & 0,77 & 0,10 & 1888 & $9,46 \mathrm{E}-10$ & $3,37 \mathrm{E}-04$ \\
\hline
\end{tabular}




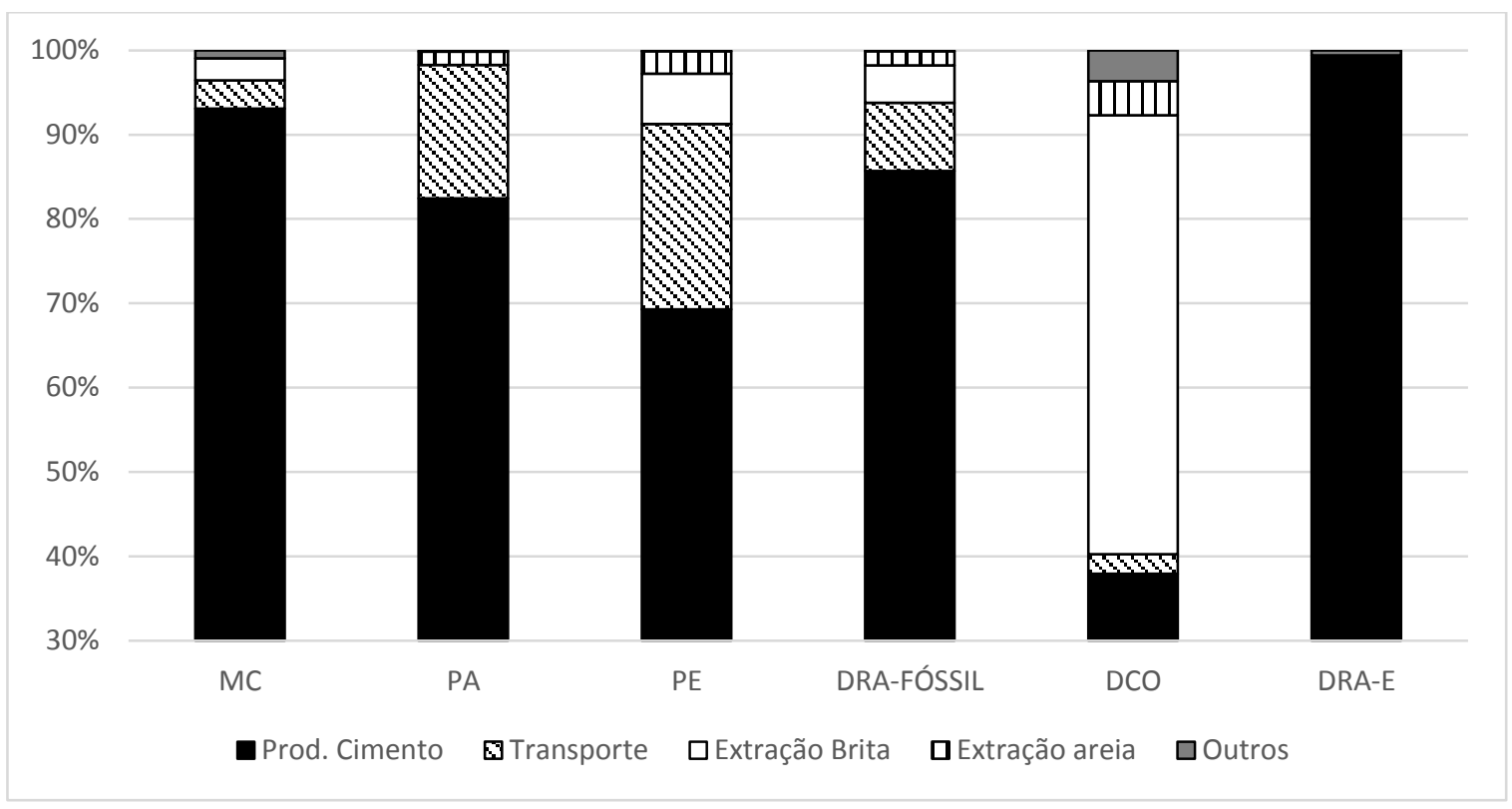

Figura 4: Participação de cada unidade de processos avaliada nos impactos do ciclo de vida do concreto 0R. Impactos avaliados: mudança climática (MC), acidificação do solo e da água (PA), eutrofização (PE), ozônio fotoquímico (DCO) e depleção de recursos abióticos fósseis (DRA-F) não fósseis (DRA-E).

O potencial de acidificação apresenta uma tendência similar, com os valores de impacto relacionados ao consumo de cimento dos concretos avaliados (participação de $82 \%$ para o traço 0R). Destaca-se também a participação do somatório de todos os modais de transporte (16\%), e da extração da areia (2\%). Neste caso, para cada um dos traços avaliados, observa-se um pequeno aumento do potencial de acidificação (entre $1 \mathrm{e}$ $2 \%$ ) nos concretos com o uso de ágata em comparação aos concretos referência, devido às emissões de NOx e $\mathrm{SO}_{2}$ no transporte rodoviário do resíduo.

No caso do potencial de eutrofização, a participação do processo de produção do cimento é ligeiramente inferior (69\%), com maior participação dos modais de transporte (22\%), pelas mesmas razões observadas nas argamassas. Salienta-se, ainda, a participação da extração da brita (entre 6\%) e da areia (3\%).

O consumo de combustíveis fósseis para a produção de $1 \mathrm{~m}^{3}$ de concreto situa-se entre 1872 e 3208 MJ. Destes, 2744 MJ (86\%) são consumidos na produção do cimento, para o traço 0R. Destacam-se ainda as participações da extração de brita (4\%) e areia (2\%), além do transporte (8\%).

Finalmente, para o impacto de depleção da camada de ozônio, a tendência é diferente das demais categorias de impacto. Nesse caso, o processo mais impactante é a extração da brita, (52\%). Esta contribuição é devida à emissão de compostos halogenados ao ar durante a produção do material. A contribuição do processo de produção do cimento é de $38 \%$, e também está relacionada a emissões atmosféricas de compostos halogenados. A participação da extração da areia é de $4 \%$, e o transporte $2 \%$.

Ainda, quando é avaliada a depleção dos recursos abióticos-elementos, novamente predomina a produção do cimento como grande consumidor de recursos, pelo uso da gipsita (Figura 4). Cabe destacar, novamente, que a extração do calcário e da areia não está contemplada na ferramenta de impacto [38].

Uma vez que as argamassas e os concretos avaliados possuem diferentes comportamentos mecânicos, os mesmos não são diretamente comparáveis por desempenharem diferentes funções. Para realizar a comparação entre o comportamento mecânico e os impactos ambientais dos materiais estudados, considerou-se que a metodologia de impacto de mudanças climáticas é representativa, por sua importância e por possuir tendência similar às demais categorias.

\subsection{Comparação entre impactos ambientais e comportamento mecânico}

Observa-se, na Figura 5, que o comportamento mecânico das argamassas estudadas está diretamente relacionado ao impacto de mudança climática, o que é esperado, uma vez que a resistência mecânica é função da relação a/c. Porém, também é possível observar que, em distintos intervalos de resistência, são encontrados valores variáveis de mudança climática. Por exemplo, para se obter resistências em torno dos $30 \mathrm{MPa}$, é possível utilizar um teor de substituição de 10\% (10R), sem comprometimento dos valores de resistência, e com 
redução na emissão de gases do efeito estufa (GEE) de 10\% (em comparação ao traço 0R). Para a faixa dos $20 \mathrm{MPa}$, é possível aplicar as argamassas com 20\% de substituição (20M), e redução de $20 \%$ na emissão de GEE (comparando as argamassas 0M e 20M). Ainda, nesta faixa, cabe destacar que a substituição de $10 \%$ $(10 \mathrm{M})$ apresentou um incremento de resistência em relação ao teor referência $(0 \mathrm{M})$, apresentando redução das emissões de $10 \%$ e acréscimo de resistência de $20 \%$, possivelmente graças ao efeito filler do pó de ágata e algum efeito pozolânico, já que nos ensaios realizados de índice de pozolanicidade, o pó obteve resultados ligeiramente inferiores ao que preconiza a NBR 12653 [27]. Finalmente, quando são requeridas resistências mais baixas (5 MPa), é possível se utilizar traços pobres em cimento com elevados teores de substituição por pó de ágata (20P, 30P), e reduzido impacto ambiental.

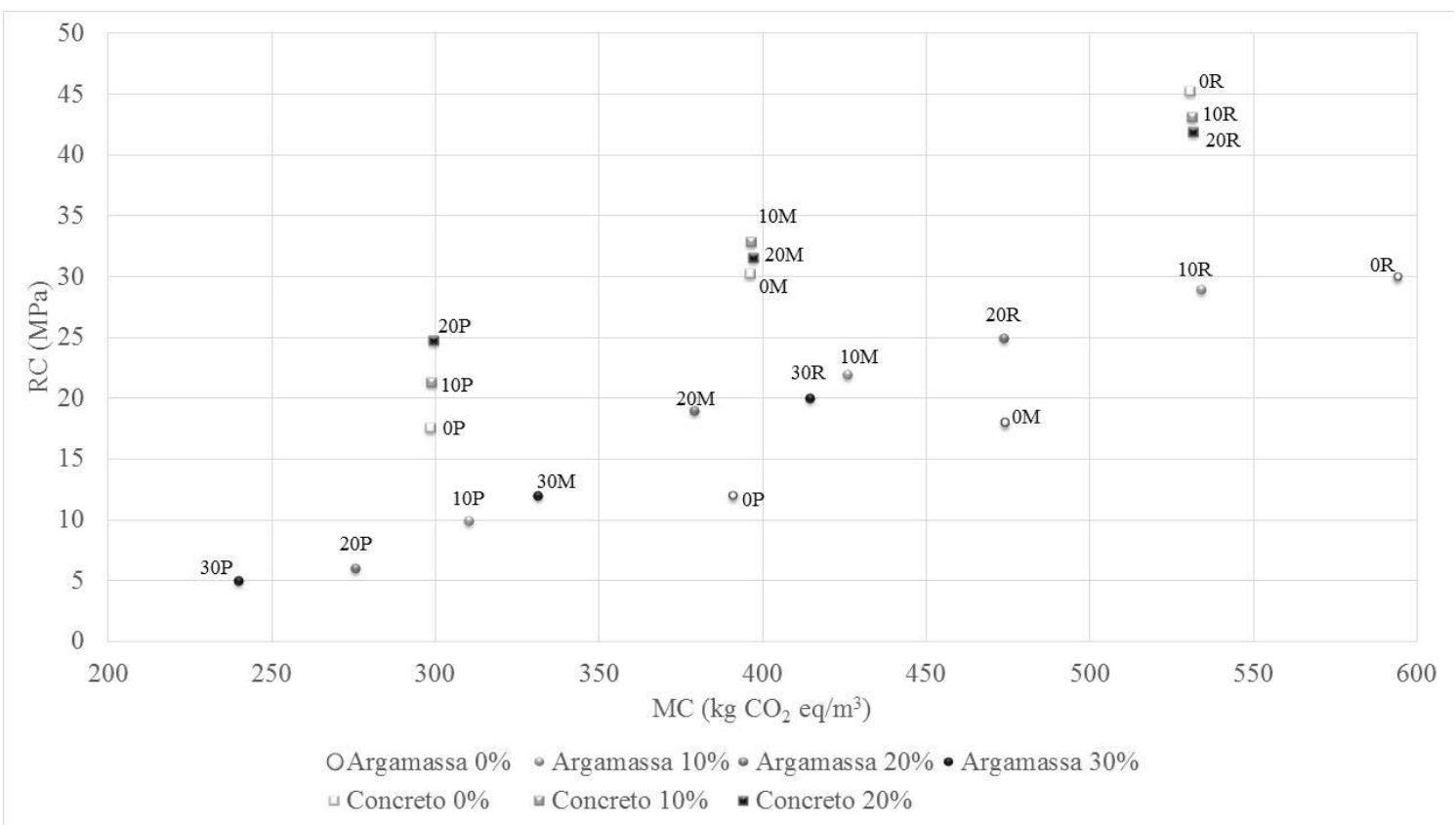

Figura 5: Comparação entre os resultados obtidos de Mudança Climática ( $\mathrm{MC}$, em $\mathrm{kg} \mathrm{CO}_{2}$-eq m $\left.\mathrm{m}^{-3}\right)$ e Resistência à compressão ( $\mathrm{RC}$, em $\mathrm{MPa}$ ) para as argamassas e concretos produzidos com diferentes níveis (\%) de substituição

Diferentemente do que ocorre nas argamassas, os concretos desenvolvidos possuem impactos similares para cada um dos traços avaliados (R, M, P), por possuírem mesmo consumo de cimento (Figura 5). Cabe destacar que as tendências das resistências à compressão são diferentes para cada um dos traços avaliados.

No caso dos concretos ricos em cimento $(\mathrm{R})$, observa-se uma redução na resistência à compressão axial (0R, 10R, 20R, Figura 5), com o aumento do teor de substituição. Os concretos de traço intermediário (M) possuem variabilidade aparentemente menor nos resultados. Para o traço pobre (P), com menor consumo de cimento, o aumento do percentual do pó de ágata nos concretos pode haver promovido um incremento na resistência à compressão axial. As misturas $10 \mathrm{P}$ e $20 \mathrm{P}$ apresentaram acréscimos de $21 \%$ e $41 \%$ em relação à mistura 0P e de $16 \%$ entre si, para um mesmo valor de emissão de gases do efeito estufa (Figura 5). Neste caso, o emprego do pó de ágata mostra-se positivo nos concretos de traço pobre, graças provavelmente ao efeito filer, aumentando a resistência à compressão sem aumentar a emissão de GEE.

\section{CONCLUSÕES}

O presente estudo realizou uma avaliação dos impactos ambientais potenciais associados ao ciclo de vida de argamassas e concretos produzidos em estudos anteriores com a aplicação do resíduo pó de rolagem de ágata em diferentes teores de substituição. Ainda que o objetivo do estudo não seja a comparação direta dos impactos ambientais dos materiais produzidos, uma vez que estes desempenham diferentes funções em termos de suas propriedades mecânicas, percebe-se a importância da realização deste tipo de estudo antes da definição dos proporcionamentos, como técnica de suporte à tomada de decisão a fim de produzir materiais de reduzido impacto ambiental.

Os resultados apresentados confirmam o potencial de aplicação do resíduo pó de rolagem de ágata na produção de concretos e argamassas. Uma vez que todos os impactos avaliados estão fortemente relacionados 
ao consumo de cimento, considera-se que o uso do resíduo em substituição ao cimento é a alternativa mais ambientalmente viável, para os casos avaliados. A participação dos processos relacionados ao transporte de mercadorias em todas as categorias de impacto valida a utilização de produtos produzidos/extraídos localmente para a redução de impactos ambientais. Especificamente para o impacto de depleção da camada de ozônio em concretos, sugere-se a utilização de resíduos em substituição ao agregado graúdo, com vistas à redução deste impacto, desde que mantidas as propriedades mecânicas dos materiais produzidos.

Finalmente, estudos futuros devem considerar a aplicação deste resíduo em substituição ao cimento Portland para a produção de concretos, além de avaliar a durabilidade dos materiais produzidos, uma vez que a redução dos impactos avaliados somente será efetiva se os materiais produzidos a partir de resíduos possuírem desempenho similar aos materiais referência.

\section{AGRADECIMENTOS}

Os autores agradecem o aporte financeiro do Conselho Nacional de Desenvolvimento Científico e Tecnológico (CNPq), Coordenação de Aperfeiçoamento de Pessoal de Nível Superior (CAPES) e Fundação de Amparo à Pesquisa do Estado do Rio Grande do Sul (FAPERGS).

\section{BIBLIOGRAFIA}

[1] BRANCO, P., VIERO, A. I. N. I. Mercado de gemas e pedras do Rio Grande do Sul. Conselho em Revista: matérias técnicas, Porto Alegre, n. 4, p. 16-18. 2004. Disponível em: <http://www.crears.org.br/crea/pags/revista/04/index.htm>. Acesso em: 12 jun. 2011.

[2] COSTA, R. M., JORNADA, M. I. H., Arranjo Produtivo de Pedras, Gemas e Joias do Alto da Serra do Botucaraí. Relatório II. Porto Alegre: FEE, 2015. Relatório do Projeto Estudo de Aglomerações Industriais e Agroindustriais no RS. Disponível em:< http://www.fee.rs.gov.br/publicacoes/relatorios/>.

[3] BETAT, E. F. Concretos produzidos com resíduos do beneficiamento de ágata: avaliação da resistência à compressão. Dissertação de M.Sc., Departamento de Engenharia Materiais, Escola de Engenharia, Universidade Luterana do Brasil, Canoas, Brasil, 2006.

[4] VENQUIARUTO, S., OSSORIO, A., ZANINI, C., et al., Aproveitamento de Resíduos de Ágata Reciclada em Materiais Cimentícios Sustentáveis, In: HARTMANN, L. A., SILVA, J. T., DONATO, M. (Orgs.). Tecnologia e Inovação em Gemas, Joias e Mineração. 1. ed. Porto Alegre: IGeo/UFRGS, pp. 99-106, 2014.

[5] DAL BELLO, A. C., CAPPELLESSO, V. G., PETRY, N. S., et al., "Avaliação da utilização de agregado miúdo reciclado de ágata a partir da análise de seu potencial reativo pelo ensaio de reação álcali-agregado (RAA)", In: Congresso Regional de Iniciação Científica e Tecnológica em Engenharia, Passo Fundo, 2013.

[6] CHIARO, S.M.X. Reação álcali-agregado em concretos brancos com agregados miúdos reciclados de ágata. 2012.78 f., Trabalho de Conclusão, Universidade Federal do Rio Grande do Sul, Escola de Engenharia, Porto Alegre, Brasil, 2012.

[7] CHIARO, S. X., PETRY, N. S., TIECHER, F., et al., Avaliação da potencialidade de desenvolvimento da reação álcali-agregado de agregados oriundos de resíduos de ágata, In: XII Congreso Latinoamericano de Patología de la Construcción y XIV Congreso de Control de Calidad en la Construcción. Cartagena, Colombia, Out. 2013.

[8] ABREU, A.G., SILVEIRA, A.A., MIRANDA, T. "Pó de resíduos de ágata como adição mineral”, In: $V$ Congreso internacional y 19a. Reunión Técnica de la Asociación Argentina de Tecnología del Hormigón. Buenos Aires, 2012.

[9] PETRY, N. S. Uso de resíduos de ágata como agregado miúdo em argamassas de cimento Portland branco, Dissertação de M.Sc., Programa de Pós-Graduação de Engenharia Civil, Universidade Federal do Rio Grande do Sul, Brasil, 2015.

[10] HUNTZINGER, D. N., EATMON, T. D. "A life-cycle assessment of Portland cement manufacturing: comparing the traditional process with alternative technologies", Journal of Cleaner Production, v. 17, n. 7, p. 668-675, 2009.

[11] VALDERRAMA, C., et al., "Implementation of best available techniques in cement manufacturing: a life-cycle assessment study", Journal of Cleaner Production, v. 25, n. 3, p. 60-67, 2012.

[12] KORONEOS, C., DOMPROS, A. "Environmental assessment of brick production in Greece", Building and Environment, v. 42, n. 5, p. 2114-2123, 2007.

[13] ABEYSUNDRA, U.G.Y., BABEL, S., GHEEWALA, S., et al., "Environmental, economic and social analysis of materials for doors and windows in Sri Lanka”, Building and Environment, v. 42, p. 2141-2149, 
2007.

[14] NORRIS, G. A., YOST, P. A. "transparent, interactive software environment for communicating lifecycle assessment results: An application to residential windows", Journal of Industrial Ecology, v. 5, n. 4, p. 15-28, 2001.

[15] BISWAS, W. K. "Carbon footprint and embodied energy assessment of a civil works program in a residential estate of Western Australia”, The International Journal of Life Cycle Assessment, v.3, n.2, 20 dez. 2014.

[16] JÖNSSON, Å., TILLMAN, A.-M., SVENSSON, T. "Life cycle assessment of flooring materials: Case study", Building and Environment, v. 32, n. 3, pp. 245-255, 1997.

[17] NEBEL, B., ZIMMER, B., WEGENER, G. "Life cycle assessment of wood floor coverings: A representative study for the German flooring industry", International Journal of Life Cycle Assessment, v. 11, n. 3, pp. 172-182, 2006.

[18] NICOLETTI, G. M., NOTARNICOLA, B., TASSIELlI, G. "Comparative Life Cycle Assessment of flooring materials: Ceramic versus marble tiles”, Journal of Cleaner Production, v. 10, n. 3, pp. 283-296, 2002.

[19] HUIJBREgTS, M. A. J., GILIJAMSE, W., RAGAS, A.M.J., et al., "Evaluating Uncertainty in Environmental Life-Cycle Assessment. A Case Study Comparing Two Insulation Options for a Dutch OneFamily Dwelling”, Environmental Science \& Technology, v. 37, n. 11, p. 2600-2608, jun. 2003.

[20] VAN DER LUGT, P., VAN DEN DOBBELSTEEN, A. A. J. F., JANSSEN, J. J. A. “An environmental, economic and practical assessment of bamboo as a building material for supporting structures", Construction and Building Materials, v. 20, n. 9, pp. 648-656, 2006.

[21] SAADE, M. R. M., SILVA, M. G., GOMES, V. “Appropriateness of environmental impact distribution methods to model blast furnace slag recycling in cement making", Resources, Conservation and Recycling, v. 99, p. 40-47, jun. 2015.

[22] STAFFORD, F. N. et al., "Life cycle assessment of the production of cement: A Brazilian case study", Journal of Cleaner Production, v. 137, pp. 1293-1299, 2016.

[23] PASSUEllo, A. C. B., OlIVEIRA, A. F., COSTA, E. B., et al., “Aplicação da Avaliação do Ciclo de Vida na análise de impactos ambientais de materiais de construção inovadores: estudo de caso da pegada de carbono de clínqueres alternativos", Ambiente construído, v. 14, n. 4, p. 7-20, 2014.

[24] PASSUELlO, A., RODRÍGUEZ, E. D., HIRT, E., et al., "Evaluation of the potential improvement in the environmental footprint of geopolymers using waste-derived activators", J. Clean. Prod., v.166, pp.680689, 2017. DOI: https://doi.org/10.1016/j.jclepro.2017.08.007.

[25] MARQUES, V. M., GOMES, L. P., KERN, A. P. "Avaliação ambiental do ciclo de vida de telhas de fibrocimento com fibras de amianto ou com fibras de polipropileno", Ambiente Construído, v. 16, n. 1, p. 187-201, 2016.

[26] MAIA DE SOUZA, D., et al. "Comparative life cycle assessment of ceramic brick, concrete brick and cast-in-place reinforced concrete exterior walls”, Journal of Cleaner Production, v. 137, pp. 70-82, 2016.

[27] ASSOCIAÇÃO BRASILEIRA DE NORMAS TÉCNICAS. Materiais pozolânicos — Requisitos: NBR 12653:2015. Rio de Janeiro, 2015.

[28] ASSOCIAÇÃO BRASILEIRA DE NORMAS TÉCNICAS. Materiais pozolânicos - Determinação da atividade pozolânica com cal aos sete dias: NBR 5751:2015. Rio de Janeiro, 2015.

[29] ASSOCIAÇÃO BRASILEIRA DE NORMAS TÉCNICAS. Materiais pozolânicos - Materiais pozolânicos - Determinação do índice de desempenho com cimento Portland aos 28 dias: NBR 5752:2014. Rio de Janeiro, 2015.

[30] ASSOCIAÇÃO BRASILEIRA DE NORMAS TÉCNICAS. Agregados — determinação da composição granulométrica: NBR NM 248. Rio de Janeiro, 2003.

[31] ASSOCIAÇÃO BRASILEIRA DE NORMAS TÉCNICAS. Agregados para concreto - Especificação: NBR 7211. Rio de Janeiro, 2009.

[32] ASSOCIAÇÃO BRASILEIRA DE NORMAS TÉCNICAS. Agregado miúdo — Determinação da massa específica e massa específica aparente: NBR NM 52. Rio de Janeiro, 2009.

[33] PE INTERNATIONAL. GaBi 6. Software-System and Databases. Construction materials extension database XIV., 2014.

[34] BUILDING RESEARCH ESTABLISHMENT, B. R. E. Product Category Rules for Type III environ- 
mental product declaration of construction products to EN 15804:2012. Watford, UK: [s.n.].

[35] DERWENT, R. G., et al., Photochemical ozone creation potentials for organic compounds in northwest Europe calculated with a master chemical mechanism. Atmospheric Environment, v. 32, n. 14-15, p. 24292441, ago. 1998.

[36] ASSOCIAÇÃO BRASILEIRA DE NORMAS TÉCNICAS. Concreto - Ensaio de compressão de corpos-de-prova cilíndricos — Método de ensaio: NBR 5739. Rio de Janeiro, 2007.

[37] ASSOCIAÇÃO BRASILEIRA DE NORMAS TÉCNICAS. Cimento Portland - Determinação da resistência à compressão: NBR 7215. Rio de Janeiro, 1996.

[38] GUINÉE, J. B., HEIJUNGS, R., HUPPES, G. Economic allocation: examples and derived decision tree. The international journal of life cycle assessment, 2004. v. 9, n. 1, p. 23-33.

[39] KNOERI, C., SANYÉ-MENGUAL, E., ALTHAUS, H.-J. "Comparative LCA of recycled and conventional concrete for structural applications”, The International Journal of Life Cycle Assessment, v. 18, n. 5, pp. 909-918, 23 jan. 2013.

\section{ORCID}

Denise Dal Molin

Ana Passuello

Angela Masuero

Camila Mokwa Zanini

Anilé Ossorio

Ana Paula Kirchheim

Simone Dornelles Venquiaruto https://orcid.org/0000-0003-1934-7533

https://orcid.org/0000-0003-2961-7787

https://orcid.org/0000-0001-9117-8346

https://orcid.org/0000-0003-1901-6638

https://orcid.org/0000-0001-8977-5221

https://orcid.org/0000-0002-8241-0331

https://orcid.org/0000-0003-4306-6686

\section{ERRATA}

Onde se lia:

Anille Ossorio-Dominguez ${ }^{1}$

Leia-se:

Anilé Ossorio Domínguez ${ }^{1}$

Revista Matéria (2019) 24 (2) e-12382 